\title{
Bruxismo durante o Sono
}

\author{
Flávio Alóe* \\ Lílian Regina Gonçalves** \\ Alexandre Azevedo*** \\ Ricardo Castro Barbosa****
}

\begin{abstract}
RESUMO
Bruxismo durante o sono (BS) é uma parassonia caracterizada por movimentos involuntários e estereotipados com ranger dos dentes durante o sono. A prevalência do BS é igual nos dois sexos, variando de 3\% a 20\% na população geral, sendo mais comum nos jovens. O BS secundário é causado por transtornos neurológicos ou pode estar associado a transtornos primários do sono e antidepressivos inibidores seletivos da recaptação da serotonina. Já o BS primário apresenta fatores de predisposição genética ou psicológica, má oclusão dentária, disfunção leve dos gânglios da base e combinações desses fatores. Os principais sinais e sintomas do BS incluem o ruído característico de ranger dos dentes, desgaste dentário, dor local, hipertrofia dos músculos masseteres e temporais, cefaléias, disfunção da articulação temporomandibular, sono de má qualidade e sonolência diurna. O diagnóstico clínico de BS é feito por meio da história do paciente, do cônjuge e do exame odontológico. A polissonografia documenta a presença de episódios de ranger dos dentes, permitindo identificar alterações da arquitetura do sono, presença de microdespertares, abalos mioclônicos de membros inferiores, roncos e distúrbios respiratórios sonodependentes. O tratamento deve ser direcionado para os fatores etiológicos com base no BS secundário. Não existe tratamento-padrão para o BS primário, devendo este ser individualizado ao paciente. O tratamento odontológico do BS primário e secundário com placas de repouso tem como objetivo prevenir danos das estruturas orofaciais e aliviar dor craniofacial. O tratamento comportamental inclui técnicas de relaxamento, abstinência de cafeína e tabaco. O tratamento farmacológico do BS primário e secundário emprega drogas agonistas dopaminérgicas, benzodiazepínicos ansiolíticos, buspirona, hipnóticos não-benzodiazepínicos, como o zolpidem, relaxantes musculares, certos antidepressivos, como mirtazapina, nefazodona, trazodona, bupopriona e drogas antiepilépticas, como a gabapentina. Aplicações locais de toxina botulínica nos músculos masseteres e temporais podem ser utilizadas em casos de bruxismo intenso não-responsivo à terapêutica convencional.

Unitermos: Ranger de dentes, bruxismo durante o sono, tratamento comportamental e farmacológico.
\end{abstract}

\section{Introdução}

Segundo Dorland's Illustrated Medical Dictionary, a palavra bruxismo vem do grego brychein, que significa ranger dos dentes. Porém, foi Pietkiewicz em 1907 que introduziu o termo "bruxomania" pela primeira vez no vocabulário médico contemporâneo, descrevendo casos de ranger de dentes durante o dia ${ }^{1}$.

A Classificação Internacional dos Distúrbios do Sono (ICSD - 1997) define o BS como movimentos estereotipados e periódicos com ranger e/ou cerrar de dentes, decorrentes da contração rítmica dos músculos masseteres durante o sono ${ }^{2}$.
O bruxismo que ocorre durante a vigília ou bruxismo diurno (BD) e o bruxismo durante o sono (BS) são entidades clínicas diferentes que ocorrem em distintos estados de consciência (sono e vigília), com diferentes etiologias, devendo, portanto, ser diferenciadas porque necessitam de estratégias de tratamentos diferentes ${ }^{3}$.

O BD é semi-involuntário, ocorrendo principalmente em vigília com contrações episódicas da musculatura da mastigação, mais comumente com cerrar da mandíbula ou ranger de dentes; é também geralmente causado por outras condições médicas (por exemplo, neurolépticos, distonia, etc.) ${ }^{3}$.

\footnotetext{
* Médico Assistente do Centro Interdepartamental para Estudos do Sono do HC-FMUSP.

** Médica Neurologista Colaboradora da Divisão de Clínica Neurológica do HC-FMUSP.

*** Médico Psiquiatra. Pós-graduando do Departamento de Psiquiatria da FMUSP e Colaborador do Centro Interdepartamental para Estudos do Sono do HC-FMUSP.

**** Cirurgião-Dentista, LIM 23 - FMUSP, do Departamento de Otorrinolaringologia da FMRP-USP
} 


\section{Epidemiologia}

Ranger de dentes durante o sono é comum na população geral. Cerca de $85 \%$ a $90 \%$ das pessoas relatam episódios de ranger de dentes ao longo dos anos de suas vidas ${ }^{4}$. A prevalência exata de ranger de dentes na população geral é imprecisa e subestimada porque a maioria dos estudos epidemiológicos de bruxismo é baseada em populações e metodologias diferentes, a partir de relatos subjetivos ou questionários preenchidos pelos portadores e ou familiares, utilizando-se diferentes definições clínicas e sintomatológicas. Por exemplo, apenas $5 \%$ a $20 \%$ dos pacientes com BS tomam consciência dos episódios de ranger de dentes ${ }^{3}$.

A prevalência de $\mathrm{BD}$, segundo relatos dos portadores, é de aproximadamente $20 \%$ da população adulta, sendo predominante em mulheres. Por outro lado, o BS acomete $14 \%$ a $20 \%$ das crianças abaixo de 11 $\operatorname{anos}^{4,5,6,7}$, sendo sua prevalência fortemente dependente da idade, aparecendo na infância após a erupção dos dentes decíduos ou no início da adolescência, reduzindo-se após os 40 anos de idade. O predomínio de BS é de $13 \%$ entre 18 e 29 anos, caindo para $3 \%$ em indivíduos de 60 anos ou mais ${ }^{4,8}$.

Um estudo franco-canadense de 1994, com 2.020 adultos, descreve BS com episódios freqüentes, ocorrendo em $5 \%$ a $8 \%$ dos adultos sem diferença entre os sexos ${ }^{9}$.

O estudo epidemiológico de Ohayon et al. realizado em 2001 com 13.057 entrevistas por telefone com pessoas da Alemanha, do Reino Unido e da Itália comprovou que BS ocorrendo pelo menos duas vezes por semana tem uma prevalência de $8,20 \%$, com taxas mais altas dos 19 até os 44 anos, mas igualmente entre os $\operatorname{sexos}^{10}$.

Estudos brasileiros de prevalência de parassonia, realizados por Braz et al. em 1990 e Palma et al. em 1995 em mil habitantes da cidade de São Paulo, registraram taxas de $6 \%$ a $8 \%$ para queixas de ranger de dentes ${ }^{11,12}$.

O BS parece ser uma doença crônica persistente com evolução a partir do seu aparecimento na infância ou na adolescência para a idade adulta. Estudos longitudinais revelam que $35 \%$ a $90 \%$ das crianças com BS evoluem com sintomas na idade adulta ${ }^{4,8}$.

Em conclusão, o BS é mais comum na infância, não é incomum na idade adulta e apresenta uma tendência para persistir na idade adulta em diante, reduzindo-se na terceira idade.

\section{Predisposição genética}

Nenhum marcador genético específico foi encontrado para o BS. Entretanto, $21 \%$ a $50 \%$ dos pacientes com
BS têm pelo menos um membro direto da família com BS. Filhos de portadores de BS apresentam maior risco de desenvolvê-lo. Estudos realizados com gêmeos monozigóticos e dizigóticos mostraram que BS é mais comum em gêmeos monozigóticos ${ }^{4,13}$.

\section{Etiologia, classificação e fatores de risco}

O BS é classificado como primário quando não há causa médica evidente, sistêmica ou psiquiátrica. É classificado como secundário quando vem associado a um transtorno clínico, neurológico ou psiquiátrico, relacionado a fatores iatrogênicos (uso ou retirada de substâncias ou medicamentos) ou a outro transtorno do sono (Tabela 1).

A maioria dos casos de BS é de etiologia primária. Bruxismo secundário pode estar associado a outros distúrbios do movimento (doença de Parkinson, doença de Huntington, síndrome de Shy-Drager, distonia oromandibular, discinesia oral tardia, síndrome de Gilles de la Tourette, espasmos hemifaciais, acatisia ou distonia tardia) ou à hemorragia cerebelar, atrofia olivopontocerebelar, nas demências, na fibromialgia, na dor miofacial, em crianças com retardo mental, com hiperatividade e déficit de atenção, na síndrome de Rett, na esquizofrenia, no transtorno do estresse pós-traumático e na bulimia nervosa $a^{3,9,13,14}$

O BS pode ser causado por uso de substâncias, como álcool, cafeína (doses altas), cocaína, anfetaminas, substâncias relacionadas às anfetaminas, como o metilenodioximetanfetamina (MDMA) ou ecstasy, assim como por medicamentos antipsicóticos antagonistas da neurotransmissão dopaminérgica, antidepressivos inibidores seletivos da recaptação da serotonina (fluoxetina, sertralina, paroxetina, citalopram, escitalopram, venlafaxina), fenfluramina e inibidores dos canais de cálcio (flunarizina) ${ }^{13}$.

Vários fatores de risco e doenças estão associados ao BS. Fumantes apresentam um risco aumentado em duas vezes de desenvolverem BS, e os fumantes que já são portadores de BS apresentam mais episódios de ranger de dentes durante o sono ${ }^{15}$.

O BS está associado com a síndrome das pernas inquietas e com os movimentos periódicos dos membros (mioclonias noturnas) em $10 \%$ dos $\operatorname{casos}^{9,13}$.

Cerca de 3,5\% a 5\% dos portadores da síndrome da apnéia-hipopnéia obstrutiva do sono apresentam $\mathrm{BS}^{10,13,16}$.

O BS pode representar uma manifestação subclínica e precoce do distúrbio comportamental do sono REM $^{17,18}$. 
Tabela 1 Classificação do bruxismo durante o sono

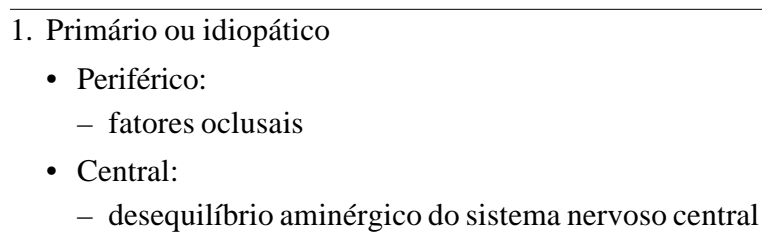

2. Secundário

- Associado a medicamentos ou outras substâncias:

- antidepressivos inibidores seletivos recaptação da serotonina

- inibidores dos canais de cálcio (flunarizina)

- levodopa

- drogas antidopaminérgicas

- antipsicóticos (antagonistas da neurotransmissão dopaminérgica)

- anfetaminas

- substâncias relacionadas às anfetaminas (MDMA metilenodioximetanfetamina ou ecstasy)

- cafeína

- cocaína

- tabaco

- álcool

- Associado a transtornos do sono (segundo a ICSD 1997*):

- síndrome das pernas inquietas

- movimentos periódicos dos membros inferiores

- distúrbio comportamental do sono REM

- síndrome da apnéia-hipopnéia obstrutiva do sono

- Transtornos neurológicos

- distonia oromandibular

- doença de Huntington

- espasmo hemifacial

- doença de Parkinson

- discinesia tardia pós-neurolépticos

- síndrome de Gilles de la Tourette

- atrofia de múltiplos sistemas

- atrofia olivopontocerebelar

- demências

- hemorragia cerebelar

- dor miofacial

- retardo mental

- hiperatividade - déficit de atenção

- síndrome de Rett

- estado de coma

- encefalopata pós-anoxia

- Transtornos psiquiátricos:

- esquizofrenia

- transtorno do estresse pós-traumático

- bulimia nervosa

- Outras doenças:

- fibromialgia

- dor miofascial

- síndrome de Sjögren
Ware et al. denominaram em 1988 como bruxismo destrutivo o quadro clínico de BS com sintomas clínicos intensos cuja atividade neuromuscular oromandibular intensa e anormal ocorre predominantemente em sono REM, diferenciando-se do BS primário que ocorre predominantemente no estágio 1 e ou 2 do sono NREM ${ }^{19}$.

Segundo descrito anteriormente, existem várias maneiras de se classificar o bruxismo, como, por exemplo, de acordo com manifestações diurnas e noturnas, intensidade das manifestações clínicas e estágio do sono; o bruxismo destrutivo por exemplo, de acordo com a etiologia, pode ser classificado como primário ou secundário (Tabela 1 ).

\section{Quadro clínico}

Considerando-se que o ranger de dentes é comum na população em geral, ele pode ser considerado um fenômeno normal. Contudo, o BS passa a ser uma anormalidade quando sinais e sintomas associados ou causados pelo ranger de dentes se tornam intensos ${ }^{3}$. A freqüência dos sintomas do BS primário é muito variável, sendo possível existir, nos casos mais leves, intervalos de tempo de até semanas sem manifestações clínicas ou surtos com períodos longos de atividade que coincidam com estresse psicológico ${ }^{20}$. O BS secundário apresenta manifestações clínicas mais constantes ${ }^{3}$.

Ranger de dentes durante a vigília e durante o sono não se limita exclusivamente a surtos de atividade orofacial. Há várias outras manifestações que ocorrem simultaneamente, como automatismos mastigatórios, movimentos orofaciais (caretas, sucção), protusão lingual, chupar de dedos e até onicofagia ${ }^{21}$.

O sintoma mais importante relatado pelo portador ou pelos familiares é o ranger dos dentes com os ruídos característicos semelhantes ao atrito de "granito contra granito", que incomoda ou preocupa os parceiros ou membros da família. Curiosamente, os portadores de BS não conseguem reproduzir voluntariamente seu ruído durante a vigília. A dor é um sintoma freqüente e importante no quadro clínico do BS. Dor ou hipersensibilidade dentária a estímulos quentes ou frios, mialgia do masseter e temporal, dores de cabeça matinal ou ao longo do dia, cervicalgia, dor de garganta e dores torácica-abdominais são relatadas. Aproximadamente $40 \%$ dos pacientes com bruxismo queixam-se de dor orofacial e rigidez mandibular matinal $^{3,22,23}$. A mialgia mastigatória (ou dor local dos músculos mastigatórios) exerce um efeito inibitório sobre o ocorrência e a intensidade de ranger de dentes 
na noite subseqüente a uma noite com atividade oromandibular intensa. Essa reação, semelhante à dor pós-exercício, é um mecanismo protetivo contra o aparecimento de dano articular. A observação clínica de uma redução do ranger dentes associada à presença de dor local com características de pós-exercício é um sinal peculiar do $\mathrm{BS}^{3}$. Cicatrizes de cortes na língua podem estar presentes ${ }^{13}$.

Bader et al. relatam que 19\% dos portadores de BS apresentam hipertensão arterial sistêmica e $31 \%$ a $50 \%$ apresentam dificuldades eréteis, além de zumbido, tensão muscular, sudorese noturna e palpitações ${ }^{23}$.

Cerca de $70 \%$ a $80 \%$ das pessoas diagnosticadas com BS apresentam sintomas de sono fragmentado, sonolência excessiva diurna moderada a intensa, sono não-restaurador, despertares noturnos, sudorese ou pesadelos $3,22,23$

Dores de dente e sintomas de disfunção da articulação temporomandibular são os sintomas que levam os portadores a buscar tratamento odontológico ${ }^{13}$.

Em conclusão, o BS é uma condição crônica altamente heterogênea com um amplo espectro de intensidade. A tabela 2 resume as principais características clínicas do bruxismo.

\section{Achados odontológicos no BS}

O bruxismo é a principal causa de lesão traumática do periodonto e de hipermobilidade dentária. A totalidade dos portadores de BS apresenta sinais de desgaste dentário com facetas polidas ou exposição da dentina, mas cerca de $40 \%$ dos portadores do BS são assintomáticos. Sensibilidade dental ao calor e/ ou ao frio pode estar presente, como também trincas e fraturas em dentes e/ou restaurações são observados em alguns $\operatorname{casos}^{13}$. Indivíduos com BS apresentam maior risco de disfunção da articulação temporomandibular (ATM $)^{24}$. Disfunção da ATM é caracterizada por dor articular, estalos, crepitação ("ranger de areia dentro do ouvido") e trismus com limitação dolorosa na abertura bucal ${ }^{22}$. A palpação da musculatura mandibular e o exame visual ao fechamento mandibular para o diagnóstico da disfunção da ATM revelam dor e/ou hipertrofia muscular abaixo da curvatura zigomática ${ }^{13}$.

\section{Fisiopatologia}

Múltiplos fatores com diferentes pesos participam da fisiopatologia do BS primário. Acredita-se existir uma associação de alterações da neurotransmissão
Tabela 2 Características clínicas do bruxismo

- Relatadas durante o sono:

- ranger dos dentes ${ }^{23,89}$

- sons notados por outra pessoa

- automatismos orofaciais mastigatórios e movimentos excessivos dos lábios e da língua

- movimentos de membros inferiores ${ }^{23}$

- sudorese excessiva durante o sono $(23 \%)^{23}$

- pesadelos

- palpitações $(62 \%)^{23}$

- despertares breves durante o sono

- despertares súbitos com taquicardia

- Relatadas pelos pacientes ao acordar ou pela manhã:

- dor ou desconforto dos músculos da mandíbula $(86 \%)^{89}$

- cefaléia com localização temporal $(48 \% \text { a } 65 \%)^{89}$

- rigidez da mandíbula e redução de sua motilidade, causando dificuldade para mastigação no desejum $(40 \%)^{89}$

- redução da intensidade do ranger de dentes desencadeada por dor muscular local ${ }^{23}$

- exacerbação dos sintomas acima com tensão emocional

- hipersensibilidade dos dentes a alimentos frios ou quentes, líquidos e $\mathrm{ar}^{90}$

- sensação de boca seca ${ }^{90}$

- ferimentos em língua, lábios e bochechas ${ }^{90}$

- dores de dente 23,89

- dor de garganta $(69 \%)^{89}$

- cervicalgia $(69 \%)^{89}$

- dor torácica-abdominal $(69 \%)^{89}$

- zumbidos

- dores musculares difusas

- Sintomas relacionados ao sono $(72 \% \text { a } 80 \%)^{23,89}$ :

- sono não-restaurador

- sonolência excessiva diurna

- fadiga

- Observadas por dentistas ou médicos:

- exacerbação de doenças periodontais ${ }^{90}$

- desgaste dentário/obturações brilhantes

- abrasão e perda do esmalte dentário com exposição da dentina ${ }^{90}$

- rotura das restaurações dentárias

- dentes partidos

- redução do fluxo salivar

- hipertrofia de masseteres, temporais, pterigóides mediais e laterais

- dor à palpação dos músculos e mobilização dolorosa da articulação temporomandibular

- cortes na língua

- parotidite ou sialolitíase $e^{90}$

- gengivite

- Outros sintomas:

- traços de personalidade com propensão à ansiedade

- redução da libido (31\% a 50\% $)^{23}$

- hipertensão arterial sistêmica 
aminérgica no $\mathrm{SNC}$, fatores psicológicos, transtornos oclusais e alterações do sono na gênese do $\mathrm{BS}^{13,25,26}$.

\section{Neurotransmissores e vias neurais no brux ismo}

Não existe uma estrutura funcional e anatômica no SNC identificável como centro neural gerador específico de movimentos oromandibulares involuntários. Contudo, existem indícios da participação da neurotransmissão dopaminérgica, noradrenérgica e serotoninérgica na gênese e na modulação do bruxismo ${ }^{26,33}$.

A figura 1 representa as vias serotoninérgicas e dopaminérgicas envolvidas na atividade mastigatória normal. Os axônios dos neurônios serotoninérgicos do núcleo dorsal da rafe se projetam via trato rafetegmento para a área ventral do tegmento mesencefálico e via trato rafe-córtex para o córtex frontal, modulando os neurônios dopaminérgicos da área ventral do tegmento mesencefálico que, por sua vez, se projetam para o córtex pré-frontal via trato mesocortical, inibindo movimentos oromandibulares involuntários (Figura 1) 27,28,29.

O sistema dopaminérgico parece ser o mais importante na gênese do BS, existindo duas hipóteses compatíveis com alterações dopaminérgicas:

\section{Hipótese hiperdopaminérgica}

Pacientes com doença de Parkinson podem apresentar ranger dos dentes durante tratamento com levodopa ${ }^{13}$. É sabido que o uso crônico de drogas antidopaminérgicas causa hipersensibilidade dos receptores dopaminérgicos, podendo causar manifestações de discinesia tardia e ranger de dentes ${ }^{34}$.

Os estados hiperdopaminérgicos induzidos por anfetaminas, metilenodioximetanfetamina (MDMA) ou ecstasy e cocaína podem também causar ranger de dentes durante a vigília e o sono ${ }^{13}$.

\section{Hipótese hipodopaminérgica}

Existem relatos na literatura de que a administração de baixas doses de L-dopa exerce efeito terapêutico com atenuação dos episódios de ranger de dentes em casos de BS primário ${ }^{26,35}$.

A associação de BS com outros transtornos relacionados com alterações da transmissão dopaminérgica contribui para o entendimento do papel dopaminérgico no BS primário. Cerca de $10 \%$ a $20 \%$ dos portadores de BS apresentam sintomas de pernas inquietas com movimentos periódicos dos membros durante o sono (MPMS) ${ }^{3}$. Existem evidências mostrando uma disfunção dopaminérgica moderada pós-sináptica do striatum e pré-sináptica do putamen

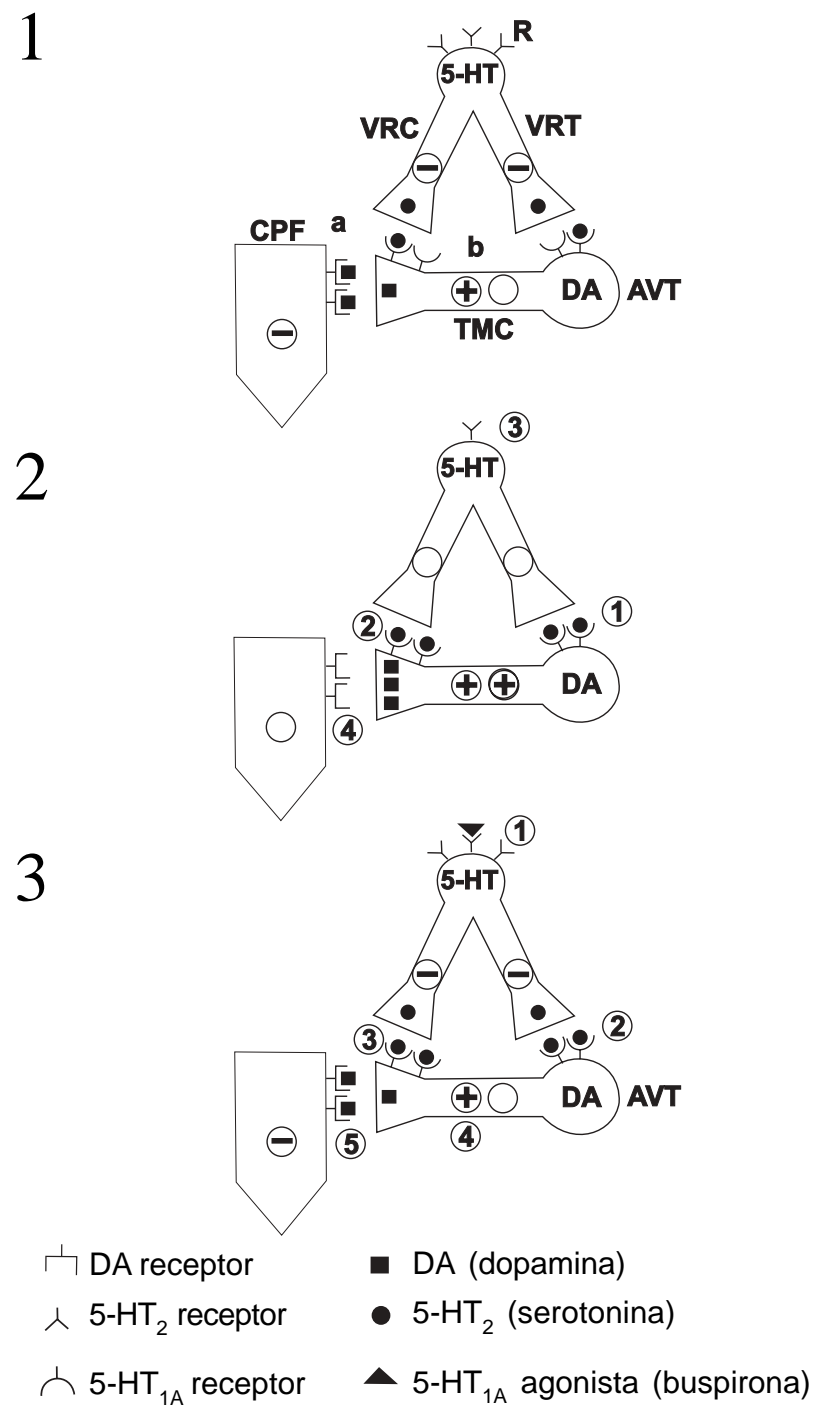

Figura 1 Efeitos da buspirona na modulação mesocortical motora.

(1) Modulação Motora Normal

Neurônios serotoninérgicos com axônios na via rafe-tegmental (VRT) e na via rafe-cortical (VRC) modulam: (a) disparos dos neurônios dopaminérgicos do trato mesocortical (TMC) originados da área ventral tegmental (VTA) e (b) a liberação sináptica de dopamina no córtex pré-frontal (CPF), respectivamente. Na rafe (R), auto-receptores 5-HT ${ }_{1 \mathrm{~A}}$ regulam disparos de neurônios serotoninérgicos. Esses mecanismos compreendem a modulação motora normal.

(2) Efeitos dos Inibidores Seletivos da Recaptação de Serotonina (ISRS) Pelo bloqueio da recaptação 5-HT, os ISRS aumentam a quantidade de serotonina disponível na fenda sináptica, levando a um aumento da ligação heterorreceptora de 5-HT (1) e diminuição dos disparos dos neurônios dopaminérgicos do trato mesocortical (TMC) (2), e down-regulation de auto-receptores $5-\mathrm{HT}_{1 \mathrm{~A}}$, aumentando os disparos de neurônios serotoninérgicos no sítio (3). O resultado é a redução da ligação de dopamina aos seus receptores (4), levando a desinibição motora pela CPF, resultando no bruxismo.

(3) Efeito da buspirona

A buspirona se liga ao receptor como um agonista de $5 \mathrm{HT}_{1 \mathrm{~A}}$ (1), causando diminuição dos disparos dos neurônios serotoninérgicos, diminuição da liberação de 5-HT e da ligação heterorreceptora (2 e 3, respectivamente). A redução da transmissão serotoninérgica causa aumento dos disparos dos neurônios dopaminérgicos (4) e aumento da liberação sináptica de dopamina (5). A modulação motora é restaurada e o bruxismo, eliminado.

Adaptado de Bostwick et $a .^{27}$. 
e caudado na gênese da síndrome das pernas inquie$\operatorname{tas}^{36,37}$. Doses baixas de levodopa e outros agonistas dopaminérgicos, como pramipexole, pergolida, lisurida, carbegolina ou bromocriptina, são efetivos no tratamento da síndrome das pernas inquietas, atestando redução da neurotransmissão dopaminérgica nos gânglios da base na síndrome das pernas inquietas $^{38,39}$. Pacientes com síndrome das pernas inquietas, de modo semelhante às pessoas com BS, apresentam resposta intensa a doses baixas de agentes dopaminérgicos; esse fato sugere que há principalmente um acometimento pré-sináptico das células produtoras de dopamina em vez de uma disfunção pós-sináptica. Pacientes com síndrome das pernas inquietas, da mesma forma que pessoas com BS, não apresentam flutuações nos sintomas motores com o tratamento a longo prazo com agentes dopaminérgicos, como ocorre com os portadores de doença de Parkinson. Isso indica que o sistema nigroestriatal não está acometido no BS e na síndrome das pernas inquietas.

Antidepressivos inibidores seletivos da recaptação de serotonina podem causar sintomas extrapiramidais, como tremores finos de membros superiores, acatisia, distonia, parkinsonismo, discinesia tardia e ranger de dentes ${ }^{40}$. O aumento da transmissão serotoninérgica induzida pelos ISRS produz uma redução dopaminérgica nos circuitos mesocortical e nigroestriatal (Figura 1), que desinibe o córtex préfrontal, causando automatismos oromandibulares e ranger de dentes durante o sono ${ }^{27,40}$. Os ISRS estão contra-indicados para o tratamento de sintomas de ansiedade ou depressão em portadores de BS.

\section{Atividade muscular mastigatória rítmica durante o sono}

Apesar de o sono ser considerado um período de relativa redução da atividade motora, há registros videopolissonográficos de atividade orofacial durante o sono ${ }^{21,30}$.

Atividade muscular mastigatória rítmica durante o sono (AMMRS) ou automatismo mastigatório é a denominação para uma atividade rítmica automática da musculatura mastigatória registrada durante o sono em pessoas normais e em portadores de $\mathrm{BS}^{30}$. Os episódios de AMMRS estimulam a salivação, e os transtornos médicos que causam xerostomia cursam com aumento de natureza compensatória do número de episódios de AMMRS $^{30}$.

Um estudo do ano de 2001 de Lavigne et al. com 82 voluntários normais demonstrou que $58 \%$ dos indivíduos avaliados apresentavam AMMRS sem ranger de dentes não associados a microdespertares e não associados com elevação fásica da freqüência cardíaca ${ }^{30}$. A constância de episódios de AMMRS declina significativamente na quarta década de vida, paralelamente com o declínio da prevalência de $\mathrm{BS}^{23,30}$.

Os episódios de AMMRS são, portanto, considerados uma manifestação motora orofacial normal ${ }^{30}$. Contudo, os portadores de BS apresentam diferenças quantitativas e qualitativas em relação à $\mathrm{AMMRS}^{31}$. A frequiência de AMMRS no BS é de três a oito vezes maior do que nos voluntários normais. Cada episódio de AMMRS em portadores de BS apresenta-se com um aumento da duração, amplitude e redução dos intervalos entre os episódios ${ }^{30}$. Além disso, os episódios de AMMRS ocorrem em associação com o ranger de dentes, sendo precedidos por microdespertares registrados e documentados no EEG e aumento fásico da frequiência cardíaca ${ }^{31}$.

O microdespertar parece ser o fator primário no desencadeamento dos episódios de AMMRS no $\mathrm{BS}^{30,31,32}$. Kato et al. demonstraram que adultos jovens portadores de bruxismo apresentaram sete vezes mais episódios de AMMRS com ranger de dentes induzidos por estímulos artificiais de despertar quando comparados com voluntários normais pareados para idade e $\operatorname{sexo}^{32}$.

$\mathrm{O}$ ranger de dentes durante o sono poderia ser considerado, portanto, uma atividade oromotora não normalmente excessiva, secundária ao microdes$\operatorname{pertar}^{30,31,32}$.

Fatores outros, como doenças, estresse e drogas, reforçariam e aumentariam a AMMRS e os surtos de ranger de dentes ${ }^{23,31}$.

\section{Aspectos psicofisiológicos}

Existe uma crença de que a ansiedade é um cofator que contribui diretamente para o BS. De fato, portadores de bruxismo parecem ser mais vulneráveis à ansiedade, ao estresse e ao desenvolvimento de sintomas psicossomáticos ${ }^{22,41}$. Ansiedade, tensão, emoções negativas e frustrações causam aumento da hiperatividade neuromuscular, redução da taxa de secreção salivar durante o sono e a vigília e conseqüentemente aumento de episódios de AMMRS e de ranger de dentes durante o sono ${ }^{22,30,42}$. A prevalência de ranger de dentes é mais elevada em pacientes adultos que vivem sob tensão emocional, que são hiperativos, agressivos ou que apresentam uma personalidade compulsiva. Verificou-se que alguns indivíduos com tensão emocional continuam a ranger os dentes mesmo após tratamento específico para a má-oclusão 
dentária, reforçando o papel da teoria comportamental no $\mathrm{BS}^{13}$.

Outros estudos empregando questionários padronizados, como o Minnesota Multiphasic Personality Inventory e o Cornell Medical Index, não demonstram diferenças nos itens de psicopatologia entre pacientes e controles normais ${ }^{43}$.

Em conclusão, pode ser que o componente comportamental seja um importante fator causal, que deve ser adequadamente avaliado e tratado quando estiver presente.

\section{Fatores odontológicos periféricos}

A teoria odontológica da década de 1960, que relatava que os elementos "periféricos" como a máoclusão dentária e os fatores mecânicos dentários seriam as bases do BS, é atualmente, no mínimo, controversa e inconclusiva ${ }^{44}$.

Pessoas sem dentes também apresentam registro de AMMRS. Isso indica que contato de superfícies dentárias não é um fator causal para o desencadeamento de atividade oromotora durante o sono $^{44}$. O estudo de Lobbezoo et al. de 2001 não documentou correlações entre alterações oclusais e atividade eletromiográfica mastigatória em pessoas com BS moderado a grave, dando a entender que os fatores periféricos não são instrumentais na gênese do $\mathrm{BS}^{45}$.

Por fim, o contato mecânico dentário é o último evento na seqüência de eventos no BS.

O primeiro evento na seqüência é a ativação do SNC com despertar e atividade alfa no EEG, ativação do SNA com aumento da frequiência cardíaca e, por último, ativação da musculatura mastigatória e contato de superfícies dentárias ${ }^{31}$.

Desse modo, não há evidências convincentes de que a teoria do desajuste oclusal seja válida ou instrumental.

\section{Manifestações oromandibulares durante 0 sono}

A maioria (60\% a $80 \%)$ dos episódios de ranger de dentes ocorre nos estágios 1 e 2 do sono NREM e raramente em sono profundo ${ }^{21,25}$. Episódios de ranger de dentes podem ocorrer mais freqüentemente ou exclusivamente durante o sono REM, como no bruxismo destrutivo ${ }^{19,23}$.

\section{Macroarquitetura do sono}

A macroarquitetura do sono de portadores de BS abaixo de 40 anos é geralmente normal, isto é, as latências de sono, as quantidades dos diferentes estágios de sono e o número de despertares não-transitórios são normais ${ }^{25,46}$. O BS em populações de mais idade é geralmente acompanhado de queixas de má qualidade de sono $^{44}$. Contudo, alguns autores relatam alterações, como aumento do número de mudanças dos estágios do sono, alterações nas latências de sono REM (prolongada ou reduzida) e redução da quantidade de sono REM $^{21,23,47}$.

\section{Alterações da microarquitetura do sono e atividade do sistema nervoso autonômico}

A freqüência de microdespertares em adultos jovens portadores de BS é geralmente normal ${ }^{30,31,50,32}$. Contudo, o aumento do número de microdespertares seguidos ou não de mudanças de estágio de sono, surtos breves de atividade alfa no EEG de sono, aumentos fásicos da freqüência cardiorrespiratória $\mathrm{e}$ pressão arterial estão freqüentemente associados a episódios de AMMRS com ranger de dentes em portadores de BS acima de 40 anos de idade $3,30,31$.

Cerca de 50\% dos episódios individuais de ranger dos dentes são precedidos de surtos breves de atividade alfa no EEG da polissonografia, observados principalmente durante o sono leve (estágios 1 e 2) e menos freqüentemente durante o sono delta e o sono $\mathrm{REM}^{23,25}$. Cerca de $86 \%$ dos episódios de ranger de dentes em adultos jovens ocorrem durante o estágio 2 do sono NREM ${ }^{32}$.

Esses surtos de atividade alfa são breves demais para serem estagiados como despertares de acordo com as regras tradicionais de estagiamento da macroarquitetura do sono $^{48}$. Por outro lado, esses despertares breves ocorrem associados com ativação do SNA simpático e causam fragmentação do sono, sintomas subjetivos de sono de má qualidade, sonolência excessiva diurna e fadiga ${ }^{49}$.

\section{Atividade do sistema nervoso autônomo em portadores de BS}

Apesar de os portadores de BS poderem apresentar sintomas autonômicos durante o sono, não há evidências de uma disfunção primária no funcionamento do SNA durante o sono ou vigília ${ }^{3}$. Por outro lado, o registro de sono de portadores de BS documenta ativação fásica e transitória do sistema nervoso autonômico simpático associado a episódios de AMMRS com ranger de dentes $\mathrm{s}^{3,30,31,32}$. Há surtos de aumento da freqüência cardíaca e da pressão arterial que podem ocorrer na ausência de despertares no EEG de sono $^{23,25,50}$. Esses episódios fásicos de ativação do sistema nervoso autonômico simpático sem des- 
pertares característicos no EEG são considerados por alguns autores como despertares autonômicos ou despertares subcorticais ${ }^{51,52}$. Os microdespertares resultam da ativação de regiões da substância reticular do tronco cerebral envolvendo circuitos aminérgicos (orexina e dopamina) controladores da atividade do SNA simpático, tálamo-corticais (despertares no EEG de sono) e circuitos motores (bruxismo e mioclonias noturnas) ${ }^{32}$. Esses despertares subcorticais são relevantes para a homeostase cardiovascular ${ }^{52,53}$.

Estudos da microarquitetura do sono que utilizam o sistema de estagiamento de padrão cíclico alternante (cyclic alternating pattern - CAP) mostram que $60 \%$ a $88 \%$ dos episódios de bruxismo que ocorrem durante o sono não-REM estão associados à fase ativa do padrão de alternância cíclica, dando a entender que a ativação do SNA está envolvida no BS ${ }^{25,54}$.

Essas alterações descritas anteriormente sugerem que os episódios de ranger de dentes são parte integrante e dinâmica de uma reação de despertar, por estarem associados a manifestações transitórias autonômicas e motoras ${ }^{23,25,50}$.

\section{Outras manifestações durante o sono}

Cerca de $60 \%$ a $80 \%$ dos episódios de ranger de dentes se associam com atividade eletromiográfica anormal e periódica dos músculos tibiais anteriores, característica da síndrome dos movimentos periódicos dos membros durante o sono (MPMS) ${ }^{19,25,55}$.

\section{Diagnóstico}

O diagnóstico de BS não é simples. De acordo com a Classificação Internacional dos Distúrbios do Sono (ICSD), bruxismo durante o sono pode ser diagnosticado clinicamente quando houver os seguintes sinais: desgaste dentário anormal, ruídos de ranger de dentes durante o sono e desconforto muscular mandibular ${ }^{2}$.

Contudo, o diagnóstico clínico de BS realizado no consultório médico por meio da anamnese apresenta sérias limitações e deve ser complementado com exame orofacial e avaliação odontológica ${ }^{21}$.

Sete sintomas e sinais clínicos auxiliam o diagnóstico de bruxismo:

1) ruído durante o ranger dos dentes;

2) queixa de dor e fraqueza nos músculos da mastigação e cefaléias ocasionais (músculos temporais);
3) desgaste dentário (um ou mais dentes);

4) sensibilidade dos dentes para frio e/ou calor;

5) hipertrofia de músculos masseteres e temporais;

6) crepitação da articulação temporomandibular ou redução da amplitude de abertura da mandíbula;

7) cicatrizes de cortes na língua ${ }^{13}$.

\section{Polissonografia}

O registro polissonográfico de uma pessoa com histórico clínico de ranger de dentes deverá incluir eletrodos de eletromiografia colocados nas regiões dos músculos masseteres, temporais, frontais bilaterais e registro audiovisual simultâneo. $\mathrm{O}$ registro audiovisual é importante para diferenciar episódios de bruxismo com o engolir e o ronco e para registrar movimentos do corpo e sons de ranger dos dentes ${ }^{21}$.

A polissonografia de portadores de BS exclui outros distúrbios do sono, tais como: síndrome da apnéia do sono, movimentos periódicos dos membros, distúrbio comportamental do sono REM e outras atividades ${ }^{13}$.

A variabilidade da ocorrência de atividades oromotoras durante o sono é mais intensa em portadores de BS leves ou moderados, fazendo-se necessárias duas noites de polissonografia, enquanto para os portadores de BS grave com menor variabilidade uma noite é suficiente ${ }^{20}$.

\section{Tratamento do BS}

Não existe atualmente uma estratégia específica, tratamento único ou sequer cura para o BS. Utilizase, portanto, tratamento comportamental, odontológico, farmacológico e suas combinações, de acordo com o perfil do portador para alívio dos sintomas. No passado recente, o tratamento do BS primário tinha como objetivo prevenir danos das estruturas orofaciais e das queixas dolorosas, mas, atualmente, o tratamento do BS primário é baseado nos mecanismos fisiopatológicos subjacentes da doença. O tratamento de $\mathrm{BS}$ secundário deverá ser dirigido à causa específica, além das medidas descritas abaixo quando também indicadas ${ }^{3}$.

\section{Tratamento comportamental}

O tratamento comportamental inclui medidas de higiene do sono, biofeedback, relaxamento, hipnoterapia e técnicas para controle de estresse ${ }^{13,56}$. 


\section{Higiene do sono}

Higiene do sono é um conjunto de instruções com o objetivo de corrigir alguns hábitos pessoais e fatores ambientais que interferem na qualidade do sono ${ }^{57}$.

\section{Higiene de sono}

1) Deite-se quando estiver sentindo sono.

2) Evite café, chá, chocolate e medicamentos com cafeína.

3) Evite álcool, no mínimo, 6 horas antes de dormir.

4) Evite fumar, no mínimo, 6 horas antes de dormir.

5) Evite comer, fumar e álcool no meio da noite.

6) Evite refeições pesadas antes de dormir.

7) Faça exercícios físicos 4 a 6 horas antes de deitar-se.

8) Reserve 20 a 30 minutos do seu tempo à noite, 4 horas antes de dormir, para "resolver" seus problemas.

9) Ingira um lanche com leite e/ou derivados e carboidratos antes de dormir.

10) Manter horários constantes para dormir e acordar, mesmo nos fins de semana.

\section{Biofeedback}

O biofeedback é uma técnica de relaxamento auxiliada pela monitorização de determinadas variáveis fisiológicas, como a eletromiografia, a temperatura cutânea, a freqüência cardíaca, a pressão arterial e a atividade eletrodérmica. $O$ paciente recebe um treinamento especializado com o objetivo de aprender a relaxar a partir da observação e controlando essas funções fisiológicas monitorizadas com o equipamento.

Os efeitos benéficos do biofeedback para tratamento do BS não persistem após o término do tratamento ${ }^{13,58}$.

\section{Técnicas de relaxamento específicas}

Incluem manobras específicas para o relaxamento da musculatura mandibular, como, por exemplo, relaxar suas mandíbulas, enquanto os lábios estão fechados e os dentes separados, várias vezes ao dia ou em que o paciente cerra voluntariamente os dentes por cinco segundos e então relaxa a mandíbula por cinco segundos, repetindo esse exercício em cinco séries, seis vezes por dia, por duas semanas ${ }^{56,58}$.

Deitar em decúbito lateral pode produzir resultados positivos com a mandíbula e o pescoço em repouso ${ }^{56}$.

\section{Hipnoterapia}

A hipnoterapia ou auto-hipnose é uma técnica de relaxamento. A pessoa relaxa os músculos da mandí- bula e associa o estado de relaxamento muscular com imagens. Em estudo realizado por Clarke et al. com oito indivíduos portadores de BS, a hipnoterapia produziu melhoras objetivas e subjetivas de até 36 meses de seguimento ${ }^{59}$.

\section{Tratamento comportamental da ansiedade}

O estresse deve ser considerado um fator permissivo associado ao BS, apesar de não existirem estudos controlados que determinem o verdadeiro papel da ansiedade no desenvolvimento e na manutenção de $\mathrm{BS}^{3}$. Manejo do estresse e mudanças no estilo de vida têm sido sugeridos se o portador de BS apresenta sintomas de ansiedade ${ }^{56}$.

\section{Tratamento odontológico}

Quatro tipos de tratamento odontológicos são recomendados $^{3}$ :

1) ajuste oclusal;

2) restauro das superfícies dentárias com colocação de coroas, pontes, etc.;

3) ortodontia;

4) uso de dispositivos intra-orais (placas).

Os três primeiros tratamentos são irreversíveis e devem ser indicados criteriosamente segundo a necessidade ${ }^{3}$.

Os dispositivos intra-orais têm como objetivos o alívio da dor e a prevenção de lesões nas estruturas orofaciais e na disfunção da articulação temporomandibular.

Dois tipos de aparelhos podem ser utilizados: os protetores bucais flexíveis ou os rígidos. O protetor bucal flexível é recomendado apenas para uso por pouco tempo devido à rápida degradação do material, enquanto as placas rígidas de estabilização são mais indicadas para uso a longo prazo ${ }^{3}$. O mecanismo de ação dos aparelhos intra-orais e sua eficiência clínica na atividade neuromuscular durante o sono e a arquitetura do sono ainda não estão bem estabelecidos ${ }^{3}$.

Alguns trabalhos mostraram redução nos níveis de atividade eletromiográfica (EMG) no BS com o uso a curto prazo da placa macia. Ao contrário, outros estudos documentam um aumento na atividade muscular em $20 \%$ dos usuários de placas rígidas e $50 \%$ dos usuários de placas flexíveis ${ }^{60}$.

A aderência do tratamento com placas a longo prazo é baixa, com menos de $20 \%$ dos pacientes usando após um ano ${ }^{61}$. 


\section{Tratamento farmacológico}

Não há um tratamento farmacológico específico e reconhecidamente efetivo a longo prazo para o BS primário e para o BD. Diferentes drogas já foram sugeridas para o tratamento farmacológico, mas existem apenas alguns poucos estudos controlados que avaliam eficácia, segurança farmacológica e repercussões sobre o $\mathrm{BS}^{13,44}$ (Tabela 3 ).

Tabela 3 Medicações que podem influenciar o bruxismo do sono

\begin{tabular}{lc}
\hline Medicamentos & Eficácia \\
- Relaxantes musculares, sedativos & \\
e ansiolíticos: & \\
- diazepam & Redução \\
- lorazepam & $?$ \\
- clonazepam & $?$ \\
- metocarbamol (relaxante muscular) & Redução \\
- Agentes dopaminérgicos: & \\
- L-dopa & Redução? \\
- bromocriptina & Sem efeito? \\
- pramipexol & $?$ \\
- pergolida & $?$ \\
- Agonistas beta-adrenérgicos: & \\
- propranolol & Redução \\
- Antidepressivos: & \\
- amitriptilina & Sem efeito \\
- fluoxetina & Exacerbação \\
- sertralina & Exacerbação \\
- paroxetina & Exacerbação \\
- venlafaxina & Exacerbação \\
- buspirona & Exacerbação \\
Toxina botulínica A & $?$ \\
\hline Adaptado de Kato et al. & \\
\hline
\end{tabular}

Adaptado de Kato et al. ${ }^{44}$.

\section{Relaxantes musculares}

Relaxantes musculares centrais do tipo benzodiazepínicos (diazepam) produzem melhoras no BS, mas devem ser usados por um tempo limitado ${ }^{13}$.

É possível que o clonazepam seja útil no BS, pois é uma das drogas de escolha para o tratamento das mioclonias noturnas, haja vista que cerca de $60 \%$ a $80 \%$ dos portadores de BS também apresentam abalos de membros inferiores ${ }^{19,25,55,62}$.

O efeito agudo dos benzodiazepínicos com sedação, déficits cognitivos e ataxia deve ser considerado antes de iniciar o tratamento, bem como seu uso a longo prazo que expõe o paciente a riscos de tolerância e dependência ${ }^{63}$.

O metocarbamol, relaxante muscular, sedativo e miorelaxante central, também pode ser utilizado no tratamento do BS, mas o mecanismo exato de ação dessa droga é desconhecido ${ }^{13}$.

\section{Agentes dopaminérgicos}

A literatura sobre a eficácia de uso de agentes dopaminérgicos é limitada e ainda inconclusiva. $\mathrm{O}$ estudo clínico duplo-cego de Lobbezoo et al. realizado em 1997 com doses baixas de L-dopa e benserazida em portadores de BS primário demonstrou apenas um efeito atenuante de $30 \%$ dos sintomas de bruxismo em pacientes com BS primário ${ }^{26}$.

A bromocriptina, um agonista do receptor D2 dopaminérgico, foi avaliada em um estudo duplo-cego controlado com placebo para tratamento de bruxismo do sono. Na dose de 7,50 mg, a bromocriptina não reduziu a frequiência de episódios de ranger de dentes durante o sono ou a amplitude das contrações musculares do masseter avaliada pela polissonografia ${ }^{33}$.

Até o momento não existem estudos com demais agonistas dopaminérgicos (lisurida, pergolida, cabergolina, pramipexol e ropinirol).

\section{Zolpidem}

Zolpidem é um hipnótico não-benzodiazepínico agonista seletivo GABAérgico de meia-vida curta. Três estudos clínicos recentes relatam efeitos positivos do zolpidem respectivamente na doença de Parkinson, na paralisia supranuclear progressiva e na síndrome das pernas inquietas com melhora significativa dos sintomas motores nessas três condições consideradas hipodopaminérgicas ${ }^{64,65,66}$.

A razão para essa melhora na doença de Parkinson, na paralisia supranuclear progressiva e na síndrome das pernas inquietas residiria na existência de uma alta densidade de receptores de ligação ao zolpidem nos gânglios da base ${ }^{67}$. Portanto, é possível que esse efeito positivo em doenças com alterações dopaminérgicas possa também ocorrer no bruxismo durante o sono, de acordo com as evidências hipodopaminérgicas da gênese do BS (retrocitado). Além disso, o zolpidem teria também atuação positiva sobre os microdespertares que ocorrem antes da AMMRS. Contudo, esta é uma hipótese a ser testada, pois não existem estudos clínicos de tratamento de BS com zolpidem.

\section{Antidepressivos}

Antidepressivos tricíclicos têm sido recomendados de modo estritamente empírico para o tratamento do $\mathrm{BS}^{13}$. Um estudo de 1997 documenta que baixas doses de amitriptilina no BS é clinicamente ineficiente para seu tratamento ${ }^{68}$. Outro estudo cruzado e duplo-cego executado em 2001 por Raigrodski et al. com dez mulheres com BS demons- 
trou que $25 \mathrm{mg}$ de amitriptilina não reduziu estatisticamente a atividade eletromiográfica masseteriana nem aumentou o tempo total de sono ${ }^{69}$.

Ware et al. sugeriram a utilização de antidepressivos supressores de sono REM, como os tricíclicos e os inibidores da MAO no tratamento do bruxismo destrutivo ${ }^{19}$.

Antidepressivos com perfil agonista dopaminérgico, como a amineptina e a bupropiona, seriam potencialmente úteis no tratamento do BS, de acordo com as teorias de modulação dopaminérgica do $\mathrm{BS}^{27}$.

Antidepressivos com o perfil antagonista 5-HT2, como a mirtazapina, a clomipramina, a trazodona, a nefazodona e a ritanserina, são alternativas a serem consideradas no tratamento de BS. O efeito anti-5HT2 dessas drogas produz um aumento do sono de ondas lentas em voluntários normais e em pessoas com depressão ${ }^{70,71}$. Sabe-se que a atividade oromandibular anormal é menos abundante e intensa em sono profundo, portanto, o aumento da quantidade de sono delta teria um papel protetor contra o $\mathrm{BS}^{21,25}$.

Agonistas dos receptores serotoninérgicos 5-HT1 e 5-HT3 facilitam a liberação de dopamina, enquanto agonistas 5-HT2 inibem a liberação de dopamina ${ }^{72}$. Assim, o bloqueio de receptores 5-HT2 promovido por esses agentes resultaria em maior atividade dopaminérgica do trato mesocortical ${ }^{27}$ (Figura 1).

Contudo, não existem, até o momento, estudos controlados para o tratamento do BS com mirtazapina, clomipramina, trazodona, nefazodona e ritanserina.

\section{Buspirona}

Bostwick et al. relataram quatro casos de bruxismo em pacientes que faziam uso de sertralina e apresentaram alívio do BS após introdução de buspirona na dose de $10 \mathrm{mg} / \mathrm{dia}^{27}$. A buspirona produz uma redução da atividade serotoninérgica do núcleo dorsal da rafe, atuando como um agonista nos auto-receptores pré-sinápticos 5-HT1A. A estimulação desses receptores diminui a freqüência de disparos do trato rafe tegumento mesencefálico e do rafe córtex pré-frontal. Como consequiência, levará a um aumento da atividade dopaminérgica e diminuição da atividade oromandibular (Figura 1).

\section{Agentes anticonvulsivantes}

Estudos recentes têm demonstrado que algumas drogas anticonvulsivantes (DAC) mais modernas produzem maior estabilidade do sono em pacientes epilépticos e não-epilépticos ${ }^{73}$.

A gabapentina, DAC agonista GABAérgica e moduladora dos canais de sódio, promove aumento do sono delta e do sono REM em indivíduos saudáveis ${ }^{73}$.
Sabe-se que a atividade oromandibular anormal é menos abundante em sono delta, portanto, o aumento da quantidade de sono delta teria um papel protetor contra o BS ${ }^{21,23}$. A gabapentina é também usada, principalmente, como droga coadjuvante no tratamento de epilepsia parcial e vem sendo usada no tratamento de ansiedade, tremor essencial, como analgésico na dor pós-herpética e na neuropatia periférica ${ }^{74}$. Ansiedade e dor são fatores freqüentemente associados com $\mathrm{BS}^{56}$.

Brown et al. demonstraram o efeito terapêutico da gabapentina $300 \mathrm{mg}$ à noite em paciente com bruxismo secundário ao uso de venlafaxina ${ }^{75}$. A gabapentina também é usada com bons resultados clínicos em doses de $1.500 \mathrm{mg} / \mathrm{dia}$ a $2.000 \mathrm{mg} / \mathrm{dia}$ no tratamento da síndrome das pernas inquietas e nas mioclonias noturnas, condição associada em cerca de $10 \%$ dos casos de $\mathrm{BS}^{9,76}$.

Portanto, a gabapentina parece ser uma droga potencialmente útil no BS por causa da sua capacidade de aumentar o sono delta, de seus efeitos analgésicos com poucos efeitos tóxicos, por reduzir a ansiedade e exibir eficiência clínica e polissonográfica na síndrome das pernas inquietas e nas mioclonias noturnas ${ }^{76}$.

O topiramato é outra DAC com mecanismos de ação GABAérgicos, antagonismo glutamatérgico e bloqueio dos canais de sódio que produz estabilidade do sono em pacientes epilépticos ${ }^{73}$. Não existem relatos de uso de topiramato em casos de BS.

\section{Agentes beta-adrenérgicos}

O propranolol, agente beta-adrenérgico, foi relatado como efetivo no tratamento de um paciente com BS primário e efetivo em dois portadores de bruxismo iatrogênico secundário a uso agudo e crônico de antipsicóticos ${ }^{77,78}$. Não se sabe se a ação do propranolol é devida à ação sedativa indireta, ou se pode estar relacionado com a redução da atividade da formação reticular medular ascendente, inibindo os motoneurônios trigeminais provavelmente por meio de receptores beta-adrenérgicos ${ }^{79}$. $\mathrm{O}$ uso de propranolol pode causar piora de distúrbios respiratórios do sono, pesadelos e insônia ${ }^{70}$.

\section{Toxina botulínica}

A toxina botulínica do tipo A (TXB-A) é um tratamento efetivo para determinados transtornos neurológicos ${ }^{79,80}$. O mecanismo de ação da TXB-A faz-se por meio do bloqueio da liberação da acetilcolina na junção neuromuscular, gerando denervação química com paresia muscular focal ${ }^{82}$. A TXB-A foi usada para tratamento de bruxismo secundário relacionado a outros transtornos do movimento, como 
distonia cervical, espasmos oromandibulofaciais e doença de Huntington ${ }^{82,83,84,85,86}$.

O efeito clínico da TXB-A no bruxismo secundário é observado em aproximadamente dois a quatro dias após a injeção, haja vista que os efeitos benéficos duram cerca de quatro meses com redução dos sintomas diurnos e da hipertrofia muscular dos masseteres e dos temporais ${ }^{83,84,85}$. Tratamento com TXB-A é relativamente seguro, e a ocorrência de disfagia e paresia da musculatura mastigatória e facial é incomum ${ }^{44}$.

Um estudo realizado no ano 2000 relata o uso de TXB-A em 18 pessoas com ranger de dentes refratário aos demais tipos de tratamento clínico e odontológico. TXB-A nas doses de $25 \mathrm{MU}$ a 100 MU foi eficiente para eliminação dos sintomas ${ }^{82}$. Não existem estudos a longo prazo comprovando a eficácia de TXB para o tratamento de bruxismo primário ou secundário ${ }^{13}$.

\section{SUMMARY}

\section{Sleep bruxism}

Sleep bruxism (SB) is a parasomnia characterized by stereotyped jaw movements manifested by involuntary, excessive and injurious teeth grinding and clenching during sleep. SB prevalence rates varies widely ranging from $3 \%$ to $20 \%$ in the general population being more common in the young and with no relevant gender difference. Secondary SB relates to medical, neurological, psychiatric, iatrogenic, drug-induced or a combination of these factors. Primary SB pathophysiology has been associated with mild dysfunction of the basal ganglia, psychosocial and environmental factors, tooth interference, genetic influences and a combination of these factors. The main signs and symptoms of BS include grinding noises, dental wear, masseter and temporal muscle hypertrophy, local pain, temporo-mandibular joint disease, unrefereshing nocturnal sleep and daytime sleepiness. Primary SB manifestations are highly variable over time. Clinical diagnosis of primary SB is made by means of the patient's history and dental examination. Sleep studies are indicated to rule out associated sleep pathology as sleep-disordered breathing, periodic leg movements during sleep and to determine sleep-state teeth grinding. Therapeutic approaches for secondary SB consist of management of the underlying medical, psychiatric or substance-related conditions. Primary SB therapeutics encompasses several customized approaches to the subject's clinical characteristics. Dental treatment with mouth gards aims primarily at preventing oro-facial damage and pain. Behavioural treatments as alcohol, caffeine, tabacco avoidance and psychotherapy are reccomended. Pharmacologic therapy with muscle relaxants, benzodiazepines, antidepressants other than SSRIs, anti-epileptic drugs can be employed. Botulin toxin type A IM applications into mastigatory muscles stand as alternative for unresponsive cases of primary and secondary SB.

\section{Keyw ords}

Teeth grinding, sleep bruxism, behavioral and drug treatment.

\section{Referências}

1. Pietkiewicz M. La bruxomanie: memoires originaux. Rev Stomatol, 14:107-16, 1907.

2. Thorpy MJ. International classification of cleep disorders: diagnostic and coding manual, revised. Rochester, Minn: American Sleep Disorders Association, 1997, pp. 182-5.

3. Bader G, Lavigne G. Sleep bruxism: an overview of an oromandibular sleep movement disorder. Sleep Medicine Reviews, 4:27-43, 2000.

4. Hublin C, Kaprio J, Partinen M, Koskenvou M. Sleep bruxism based on a self-report in a nationwide twin cohort. J Sleep Res, 7:61-7, 1998.

5. Reding GR, Rubright WC, Zimmerman SO. Incidence of bruxism. J Dent Res, 45:1198-204, 1966.

6. Goulet JP, Lund JP, Montplaisir J. Daily clenching nocturnal bruxism and stress and their association with TMD symptoms. J Orofac Pain, 7:120, 1998.

7. Windmalm SE, Christiensen RL, Gunn SM. Oral parafunctions as temporomandibular disorder risk factors in children. J Craniomand Pract, 13:242-6, 1995.

8. Magnusson T, Carlsson GE, Engemark I. Changes in subjective symptoms of craniomandibular disorders in children and adolescents during a 10-year period. J Orof Pain, 7:76-82, 1993.

9. Lavigne GJ, Montplaisir J. Restless legs syndrome and sleep bruxism: prevalence and association among Canadians. Sleep, 17:739-43, 1994.

10. Ohayon MM, Li KK, Guilleminault C. Risk factors for sleep bruxism in the general population. Chest, 119:53-61, 2001.

11. Braz S, Hirshkowitz M, Tufik S, Neumann B. Parasomnia complaints among 1.000 residents of São Paulo. Sleep Res, 9:197, 1990.

12. Palma BD, Anderesen ML, Mello MT, Tufik S. Sleep complaints in São Paulo city: a comparision between the years 1987 and 1995. Sleep Res, 26:455, 1997.

13. Lavigne GJ, Manzini C. Bruxism. In: Kryger, MH, Roth T, Dement WC. Principles and pratice of sleep medicine. Third Edition. Philadelphia, WB Saunders, 2000, pp. 773-85.

14. Pratap-Chand R, Gourie-Devi M. Bruxism, its significance in coma. Clin Neurol Neurosurg, 87:113-7, 1985.

15. Lavigne GJ, Lobbezoo F, Rompré PH. Cigarrete smoking as risk factor or an exacerbating factor for restless legs syndrome and sleep bruxism. Sleep, 20:290-3, 1997.

16. Okeson JP, Philips BA, Berry DT. Nocturnal bruxism events in subjectcs with sleep-disordered breathing and control subjects. J Craniomandib Disord, 5:258-64, 1991.

17. Tachibana N, Yamanaka K, Kaji R et al. Sleep bruxism as a manifestation of subclinical rapid eye movement sleep behavior disorder. Sleep, 17: 555-8, 1994. 
18. Schenck C, Mahowald M. REM sleep behaviour disorder clinical, developmental, and neuroscience perspective 16 years after its formal identification in sleep. Sleep, 25 : 120-38, 2002.

19. Ware JC, Rugh JD. Destructive bruxism: sleep stages relationship. Sleep, 11:172-81, 1998.

20. Lavigne GJ, Guitard F, Rompré PH, Montplaisir JY. Variability in sleep bruxism over time. J Sleep Res, 10: 237-44, 2001.

21. Lavigne GJ, Rompré PH, Montplaisir JV. Sleep bruxism: a validity of research diagnostic criteria in a controlled polysomonographic study. J Dent Res, 75:546-52, 1996.

22. Kampe T, Tagdae T, Bader G, Edman G, Karlsson S. Reported symptons and clinical findings in a group of subjects with long standing bruxism behaviour. J Oral Rehabil, 24:581-7, 1997.

23. Bader GG, Kampe T, Tagdae T, Karlsson S, Blomqvist M. Descriptive physiological data on a sleep bruxism population. Sleep, 20:982-90, 1997.

24. Goulet JP, Lund JP, Montplaisir J. Daily clenching nocturnal bruxism and stress and their association with TMD symptoms. J Orofac Pain, 7:120, 1998.

25. Macaluso GM, Guerra P, Giovanni GD, Boselli M, Parrino L, Terzano MG. Sleep bruxism is a disorder related to periodic arousals during sleep. J Dent Res, 77:565-73, 1998.

26. Lobbezoo F, Lavigne GJ, Tanguay R, Montplaisier JY. The effect of catecholamine precursor L-dopa on sleep bruxism: a controlled clinical trial. Mov Disord, 12:73-8, 1997.

27. Bostwick JM, Jaffe MS. Buspirone as an antidote to SSRIinduced bruxism in 4 cases. J Clin Psychiatry, 60:85760, 1990.

28. Ellison JM, Stanziani P. SSRI-associated nocturnal bruxism in four patients. J Clin Psychiatry, 54:432-4, 1993.

29. Dyken ME, Rodnitzky RL. Periodic, aperiodic, and rhythmic motor disorders of sleep. Neurology, 42:68-74, 1992.

30. Lavigne GJ, Rompré PH, Poirer G, Huard H, Kato T, Montplaisir JY. Rhythmic mastigatory muscle activity during sleep in humans. J Dent Res, 80:443-8, 2001.

31. Kato T, Rompré PH, Montplaisir JY, Sessle BJ, Lavigne GJ. Sleep bruxism: an oromotor activity secondary to micro-arousal. J Dent Res, 80:1940-4, 2001.

32. Kato T, Montplaisir JY, Guitard F, Sessle BJ, Lund JP, Lavigne GJ. Evidence that experimentally induced sleep bruxism is a consequence of transiente arousals. J Dent Res, 80:1940-4, 2003

33. Lavigne GJ, Soucy JP, Lobbezoo F, Manzini C, Blanchet PJ, Montplaisis JY. Double-blind, crossover, placebocontrolled trial of bromocriptine in pacientes with sleep bruxism. Clin Neuropharmacol, 2:145-9, 2001.

34. Micheli F, Pardal MF, Gatto M. Bruxism secondary to chronic anti-dopaminergic drug exposure. Clin Neuropharmacol, 16:315-23, 1993.

35. Lobbezoo F, Sourcy JP, Montplaisir J. D2 receptor binding in sleep bruxism: a controlled study with iodine-123iodobenzamide and single photon emission computed tomografy. J Dent Res, 75:1804-10, 1996.

36. Turjanski N, Lees AJ, Brooks DJ. Striatal dopaminergic function in restless legs syndrome: 18F-dopa and 11Craclopride PET studies. Neurology, 52:932-7, 1999.
37. Ruottinen HM, Partinen M, Hublin C et al. An FDOPA PET study in patients with periodic limb movement disorder and restless legs syndrome. Neurology, 54:502-4, 2000.

38. Montplaisir J, Nicolas A, Denese R, Gomez-Mancilla B. Restless legs syndrome improved by pramipexole: $A$ double-blind randomized trial. Neurology, 52:938-43, 1999.

39. Wetter TC, Stiasny K, Winkelmann J et al. A randomized controlled study of pergolide in patients with restless legs syndrome. Neurology, 52:944-50, 1999.

40. Leo R. Movement disorders associated with the serotonin selective reuptake inhibitors. J Clin Psychiatry, 57:44954, 1996.

41. Pierce CJ, Christman K, Bennet ME et al. Stress, antecipatory stress, and psychologic measures related to bruxism. J Orofac Pain, 9:51-6, 1995.

42. Biondi M, Picardi A. Temporomandibular joint paindysfunction syndrome and bruxism: etiopathogenesis and treatment from a psychosomatic integrative viewpoint. Psychother Psychosom, 53:84, 1993.

43. Harness DM, Peltier B. Comparision of MMPI scores with self-report of sleep disturbance and bruxism in the facial pain population. J Craniomandibular Pract, 10:70-4, 1992.

44. Kato T, Thie N, Montplaisir J, Lavigne G. Bruxism and orofacial movements during sleep. Dental Clinics of North America, 45: 657-684, 2001.

45. Lobbezoo F, Rómpre PH, Soucy JP et al. Lack of association between occlusal-cephalometric measures side imbalance in striatal D2 receptors binding in sleeprelated oromotor activities. J Orofac Pain, 15:64-71, 2001.

46. Sjöholm T, Lehtinen I, Helenius H. Masseter muscle activity in diagnosed sleep bruxists compared with nonsymptomatic controls. J Sleep Res, 4:48-55, 1995.

47. Boudros NN, Montgomery MT, Nishioka G, Hatch JP. The effects of severe bruxism on sleep architecture: a preliminary report. Clin Eletroenceph, 24:59-62, 1993.

48. American Academy of Sleep Medicine. EEG arousals: Scoring rules and examples. Sleep, 15:173-194, 1992.

49. Stepannski $E$. The effect of sleep fragmentation on daytime function. Sleep, 25:268-76, 2002.

50. Okeson JP, Philips BA, Berry DTR, Baldwin RM. Nocturnal bruxing events: a report of normative data and cardiovascular response. J Oral Rehab, 21:623-30, 1994.

51. Sforza E, Nicolas MD, Lavigne G, Gosselin A, Petiti D, Montpalisir JY. EEG and cardiac activation during periodic leg movements in sleep. Neurology, 52:786-91, 1999.

52. Sforza E, Jouny C, Ibanez V. Cardiac activation during arousals in humans: further evidence for hierarchy in the arousal response. Clin Neurophysiol, 111:1611-19, 2000.

53. Weiss JW, Remsburg S, Garpestad E et al. Hemodynamic consequences of obstructive sleep apnea. Sleep, 19: 38897, 1996.

54. Zucconi M, Oldani A, Ferini-Strambi L. Arousal fluctuations in non-rapid eye movement parasomnias: the role of cyclic alternating pattern as a measure of sleep instability. $J$ Clin Neurophysiol, 12:147-54, 1995.

55. Bader G, Kampe T, Tagdae T. Body movement during sleep in subjects with long standing bruxing behavior. Int J Prosthodont, 13:327-33, 2000.

56. Thompson BA, Blount BW, Krumholz TS. Treatment Approaches to Bruxism. Am Fam Physician, 49:161722, 1994. 
57. Morin CM, Hauri P, Espie CA, Spielman AJ, Buysse DJ, Bootzin RR. Nonpharmacological treatment of chronic insomnia. Sleep, 22:1134-45, 1999.

58. Kato T, Thie N, Montplaisir J, Lavigne G. Bruxism and orofacial movements during sleep. Sleep Medicine Reviews, 4:27-43, 2000.

59. Clarke JH, Reynolds PJ. Suggestive hypnotherapy for nocturnal bruxism: a pilot study. Am J Clin Hypnosis, 33: 248-53, 1999.

60. Okeson JP. The effects of hard and soft occlusal splints for nocturnal bruxism. J AM Dent Ass, 114:788-91, 1987.

61. Yustin D, Neff P, Rieger MR, Hurst I. Characterization of 86 bruxing patients and long term study of their management with oclusal devices and other forms of therapy. J Orofacial Pain, 7:54-60, 1993.

62. Hening W, Allen R, Early $\mathrm{C}$ et al. The Treatment of Restless Legs Syndrome and Periodic Limb Movement Disorder. Sleep, 22:970-99, 1999.

63. Roehrs T, Roth T. Hypnotics: efficacy and adverse effects. In: Kryger MH; Roth, Dement WC. Principles and practice of sleep medicine. 3 ed. Philadelphia, WB Saunders, 2000 pp. 414-8.

64. Daniele A, Albanese A, Gainotti G, Gregori B, Bartolomeo P. Zolpidem in Parkinson's disease. Lancet, 349:1222-3, 1997.

65. Daniele A, Moro E, Bentivoglio AR. Zolpidem in progressive supranuclear palsy. N Engl J Med, 341:5434, 1999

66. Bezerra MLS, Matinez JVL. Zolpidem in Restless Legs Syndrome. Eur Neurol, 48:180-1, 2002.

67. Niddam R, Dubois A, Scatton B, Arbilla S, Langer SZI Autoradiographic localization of $[3 \mathrm{H}]$ zolpidem binding sites in the rat CNS: Comparison with the distribution of $[3 \mathrm{H}]$ flunitrazepam binding sites. J Neurochem, 49:890-9, 1987.

68. Mohamed SE, Christensen LV, Penchas J. A randomized double-blind clinical trial of the effect of amitriptyline on nocturnal massateric motor activity (sleep bruxism). J Craniomandibular Pract, 15:326-32, 1997.

69. Raigrodski AJ, Christensen LV, Mohamed SE, Gardine DM. The effect of four-week administration of amitriptyline on sleep bruxism. A double-blind crossover clinical study. Cranio, 19:21-5, 2001

70. Schweitzer PK. Drugs that disturb sleep and wakefulness In: Kryger, MH, Roth T, Dement WC. Principles and practice of sleep medicine. 3 ed. Philadelphia, WB Saunders, 2000, pp. 441-61.

71. Aslan S, Isik E, Cosar B. The effects of mirtazapine on sleep: a placebo controlled, double-blind study in healthy young volunteers. Sleep, 25:666-8, 2002.

72. Moreno RA, Moreno DH, Soares MBM. Psicofarmacologia de antidepressivos. Rev Bras Psiquiatria, 21: 24-40, 1999.

73. Sammaritano $M$, Sherwin A. Effect of anticonvulsants on sleep. Neurology, 54:16-24, 2000.

74. Pollacak MH, Mathews J, Scott EL. Gabapentin as a potential treatment for anxiety disorders. Am J Psychiatry, 155:992-3, 1998.
75. Brown ES, Hong SC. Antidepressant-induced bruxism sucessfully treated with gabapentin. JADA, 130:1467-9, 1999.

76. Garcia-Borroguero D, Larrosa O, Llave Y, Verger K, Masramon X, Hernandez G. Treatment of restless legs síndrome with gabapentin. Neurology, 59:1573-9, 2002.

77. Sjöholm T, Lehtinen, Piha SJ. The effect of propranolol on sleep bruxism: hypothetical considerations based on a case study. Clin Auton Res, 6:37-40, 1996.

78. Amir I, Heremesh H, Gavisg A. Bruxism secondary to antipsychotic drug exposure: a positive response to propranol. Clin Neuropharmacol, 20:86-89, 1997.

79. Lund JP. Mastication and its control by the brainstem. Crit Rev Oral Biol Med, 2:33-64, 1991

80. Jankovic J, Brin MF. Therapeutic uses of botulinum toxin N Engl J Med, 324:1186-94, 1991.

81. Singer $C$. Indications and management of botulinum toxin. Rev Neurol, 29:157-62, 1999.

82. Tan E-K, Jankovic J. Treating Severe Bruxism with Botulinum Toxin. J Am Dent Assoc, 131:211-6, 2000.

83. Watts MW, Tan E-K, Jankovic J. Bruxism and craniocervical dystonia: is there a relationship? Journal of Craneomandibular Practice, 196-201, 1999.

84. Berardelli A, Mercuri B, Priori A. Botulinum toxin for facialoral-mandibular spasms and bruxism. In: Jankovic $\mathrm{J}$ and Hallett M (ed). Neurological Disease and Therapy, Therapy with botulinum toxin. New York, Marcel Dekker, 1994, pp. 361-7.

85. Tan E-K, Jankovic J, Ondo W. Bruxism in Huntington disease. Mov Disord, 15:171-3, 2000.

86. Ivanhoe CB, Lai JM, Francisco GE. Bruxism After brain injury: sucessful treatment with botulinum toxin-A. Arch Phys Med Rehabil, 78:1272-3, 1997.

87. Lavigne GJ, Rompré PH, Guitard F, Sessle BJ, Kato T, Montplaisir JY. Lower number of K-complexes and Kalphas in sleep bruxism: a controlled quantitative study. Clin Neurophysiol, 113:686-93, 2002.

88. Lavigne GJ, Kato T, Kolta A, Sessle BJ. Neurobiological mechanisms involved in sleep bruxism. Crit Rev Oral Biol Med, 14:30-46, 2003.

89. Kampe T, Edman G, Tagdae T, Bader G, Karlsson S. Personality traits in a group of subjects with a longstanding bruxism hehaviour. J Oral Rehabil, 24:581-7, 1997.

90. Rugh JD, Harlan J. Nocturnal bruxism and temporomandibular disorders. Adv Neurol, 49:329-41, 1988.

Trabalho realizado no Centro Interdepartamental para Estudo do Sono do Hospital das Clínicas da Universidade de São Paulo (CIES HC-FMUSP)

Endereço para correspondência

Dr. Flávio Alóe

Rua Bergamota, 326/72

CEP 05468-0000 - São Paulo, SP

Tel.: (11) 3071-0972

E-mail: piero.ops@zaz.com.br 